\title{
Antenatal screening: its use in assessing obstetric risk factors in Zimbabwe
}

\footnotetext{
Department of Epidemiology, University of Washington, Seattle VD Tsu Correspondence to: Dr V D Tsu, 2532 11th Avenue West, Seattle, WA 98119 USA.

Accepted for publication September 1993
}

\begin{abstract}
Study objective - To assess the predictive utility of obstetric risk factors for identifying before the onset of labour those women at high risk of obstetric complications in a developing world setting, where home deliveries predominate and emergency transport is scarce.

Design - Risk factors derived from two population based, case-control studies (one of cephalopelvic disproportion and one of post partum haemorrhage), carried out in Zimbabwe were used to construct weighted and unweighted scores, a variety of screening algorithms, and sets of probabilities estimated from logistic regression models. These screening tests were evaluated for sensitivity, specificity, positive predictive value, and "cost" (the proportion of the population testing positive). Each complication was evaluated separately and the two were then pooled. Participants - All were Harare residents with singleton, vertex deliveries and spontaneous onset of labour. A total of 201 experienced cephalopelvic disproportion, 150 had post partum haemorrhage, and 299 had normal, unassisted deliveries.

Measurements and main results Largely because of the very low incidence of the two complications studied $(1 \%$ or less), positive predictive values were low (less than 7\%). Holding "cost" constant at $10 \%$, a screening test for cephalopelvic disproportion could predict $42.3 \%$ of cases compared with only $35.0 \%$ of those with post partum haemorrhage. Weighted scores had little advantage over unweighted ones, and probabilities from the logistic regression models did not differentiate cases from controls very well.

Conclusions - With simple algorithms based on maternal height, parity, and obstetric history, more than one third of women at risk for potentially fatal complications could be identified at relatively small cost to themselves or the health care system.
\end{abstract}

(F Epidemiol Community Health 1994;48:297-305)

One of the primary goals of antenatal care is to identify those women with raised risks for problems during pregnancy or delivery, in order to ensure that precautionary measures are instituted where possible or more intensive medical care is available should it be needed.
In the developing world, where most women deliver outside formal health care facilities and emergency transport is often difficult or impossible to obtain, the ability to identify those women who are at high risk of delivery complications and who ought, therefore, to deliver at a hospital would be very valuable. Such referrals could greatly reduce the present high levels of maternal mortality and morbidity, if women followed the recommendations and if effective hospital care were then provided.

Despite all the attention paid to identifying "high risk" women during pregnancy, there are no published studies linking risk factor identification with reduced maternal or perinatal morbidity or mortality. There are many reasons for the paucity of data on this complex inter-relationship, including lack of conceptual clarity among clinicians working in this area, multifactorial outcomes and aetiologies, logistical difficulties in implementing needed research, and ethical dilemmas.

Unlike other types of epidemiological research which look at risk factors as a means of untangling aetiologies, obstetric risk factors are often used as a screening device to assist in patient management. In this situation, a risk factor is noteworthy if it is an effective predictor of the outcome of interest, even if it is only indirectly related to the outcome and cannot itself be changed or prevented. Risk factor identification is often focussed on secondary rather than primary prevention, since many maternal complications can be treated but not prevented from occurring.

Research into obstetric risk factors has not been a high priority in industrialised countries, since most women have ready access to competent medical care there and poor maternal outcomes are relatively rare. Maternal risk factors have been studied primarily for their value in predicting or preventing unfavourable perinatal outcomes. ${ }^{1-5}$ Such research has been difficult to undertake in developing world settings, where hospital based obstetric patients are usually unrepresentative of parturient women generally, ${ }^{8}$ and data on deliveries outside hospital are quite difficult to obtain. The problems are compounded by the diversity of complications for which screening is employed and the inter-relatedness of the potential risk factors. Different components within an outcome may have different risk factors that cancel each other out when lumped together for analysis. In addition, there is a considerable time lag between the risk assessment and the outcome of interest, during which conditions can change and interventions can take place. Evaluation is complicated by the fact that the benefits and hazards of the test are difficult to 
quantify as are the consequences of being incorrectly classified.

The present study was undertaken in an effort to predict risk for two well defined complications for which the interventions have already been proved relatively effective, post partum haemorrhage and cephalopelvic disproportion. A site was chosen (Harare, Zimbabwe) that permitted nearly $90 \%$ of all local births to be represented in the study, while still being able to include data on a wide range of pertinent variables, data that were collected before the outcome was known.

\section{Methods}

Risk factors for the two complications were identified through a multivariate logistic regression analysis of data from Harare, Zimbabwe. The data came from two case-control studies involving 203 women with cephalopelvic disproportion, 151 women with post partum haemorrhage, and 299 women with normal vaginal deliveries. Details on the subjects and data collection and analysis are reported elsewhere. ${ }^{78}$ Briefly, all cases and controls were women resident in Harare with singleton, vertex deliveries and spontaneous onset of labour. Cephalopelvic disproportion cases included eligible women delivering in Harare during eight months in 1989 with an operative delivery for cephalopelvic disproportion. In addition to meeting a strict set of criteria designed to ensure that cephalopelvic disproportion was the primary cause of the operative delivery, eligible cases were rated as "definite" or "probable" by at least two of a panel of three senior obstetricians who independently reviewed the abstracted case records. Post partum haemorrhage cases occurred in the same period and included all eligible women with a recorded post partum blood loss of at least $600 \mathrm{ml}$ after a normal, unassisted vaginal delivery. Controls were matched to cases by the facility where the case originally was booked to deliver and by the week of the delivery. Case data were abstracted from the woman's medi-

Table 1 Risk factors and their relative (RR) estimates from the logistic regression models

\begin{tabular}{lll}
\hline Risk factor & $\begin{array}{l}\text { Estimate of } \\
\text { the adjusted } * \\
R R\end{array}$ & $\begin{array}{l}95 \% \\
\text { confidence } \\
\text { interval }\end{array}$ \\
\hline
\end{tabular}

\footnotetext{
Cephalopelvic disproportion: Age 35+

Height $<160 \mathrm{~cm}$

Nulliparous

Operative delivery in last pregnancy

Neonatal death in last pregnancy

Antenatal hospitalisation for PIH

Post partum haemorrhage:

Age $35+$

Low parity $(0,1)$

Poor obstetric outcome last pregnancy $\dagger$

Antenatal haemoglobin $<12 \mathrm{~g} / \mathrm{dl}$

Antenatal hospitalisation for

pregnancy related problem
}

\begin{tabular}{|c|c|}
\hline $\begin{array}{r}2 \cdot 1 \\
2 \cdot 0 \\
13 \cdot 8 \\
9 \cdot 5 \\
4 \cdot 5 \\
6 \cdot 8\end{array}$ & $\begin{array}{l}(0.96,4 \cdot 4) \\
(1 \cdot 3,3 \cdot 0) \\
(7 \cdot 3,25 \cdot 9) \\
(3 \cdot 8,23 \cdot 6) \\
(1.3,15.9) \\
(1.6,28 \cdot 3)\end{array}$ \\
\hline $\begin{array}{l}2 \cdot 6 \\
1 \cdot 7 \\
1 \cdot 8 \\
2 \cdot 2\end{array}$ & $\begin{array}{l}(1.2,5 \cdot 8) \\
(1 \cdot 1,2 \cdot 7) \\
(0.94,3 \cdot 7) \\
(0.99,5 \cdot 0)\end{array}$ \\
\hline & $(1.4,12 \cdot 8)$ \\
\hline
\end{tabular}

* Adjusted for the other factors listed and for facility booked (on which cases and controls were matched).

$\dagger$ Post partum haemorrhage, 1st or 2nd trimester, fetal death, stillbirth, neonatal death.

PIH = pregnancy induced hypertension. cal record while she was still in the hospital, while data for controls were abstracted within a few weeks after delivery from the uniform maternal record form used for all deliveries in the public system.

To evaluate the effectiveness of various factors in screening pregnant women for hospital rather than home delivery, only factors detectable before the onset of labour were used. Risk factors identified for cephalopelvic disproportion included advanced maternal age ( 35 years and older), short stature (less than $160 \mathrm{~cm}$ ), nulliparity, a history of operative delivery or neonatal death in the preceding pregnancy, and admission to hospital for pregnancy induced hypertension in the current pregnancy. Those identified for post partum haemorrhage also included advanced maternal age and low parity, but included a more general history of poor obstetric outcome in the preceding pregnancy, anaemia during the current pregnancy, and admission to hospital for any pregnancy related problem before the onset of labour as well. Table 1 lists the factors and their adjusted relative risk (RR) estimates from the two logistical models.

Several approaches were undertaken to devise screening tests using these factors and to evaluate the resulting systems. Initially, the logistic models were used to calculate estimated probabilities for each subject for the two separate outcomes (see Appendix for an explanation of the computations). In addition, a variety of different algorithms were developed using cumulative scores based on the sum of all risk factors, either unweighted or weighted by their RR values, or simple tests based on the presence of one or more selected risk factors, alone or in combination with each other. Screening tests were devised for each of the two complications separately and, since a good screening system should identify women at risk for either of these problems, for a pooled population consisting of both case groups and the controls. Factors used for screening the pooled population were derived from those elements the two case studies had in common.

Sensitivity, specificity, and likelihood ratios (the ratio of sensitivity to 1-specificity) were calculated for the dichotomous algorithms and for various cut off points for the continuous scores and the probabilities. All logical combinations of factors were tested, but only those with higher likelihood ratios were reported here. Receiver operating characteristic (ROC) curves, plots of the true positive rate (sensitivity) against the false positive rate (1-specificity), were derived for the probabilities estimated from the logistic models and for the weighted and unweighted cumulative scores.

Predictive values for a test could not be derived from this case-control study directly, but were calculated using the estimated incidences of cephalopelvic disproportion and post partum haemorrhage in this population. Only likelihood ratios and predictive values for positive tests are considered here, since negative tests in this particular setting will result in no change in the course of action planned before the test (that is, either home or hospital deliv- 
ery). The proportion of the population that would be referred according to a particular test constitutes an important measure of the "cost" of the test to the system. Like the predictive values, it was calculated using the estimated incidences of cephalopelvic disproportion and post partum haemorrhage in this population.

\section{Results}

Scores for cases and controls, based on sums of the risk factors, either unweighted or weighted by the RR values, were compared using ROC curves. For cephalopelvic disproportion (see fig 1), the score based on weighted factors performed modestly better than one using unweighted factors, but even at its optimum point (the point on the curve closest to the upper left corner), that is using a cut off of 4 or more, the weighted score had both a sensitivity and specificity of only $75 \%$. The curves for post partum haemorrhage (fig 2 ) were only a bit above the diagonal line representing results that could be expected by chance if the test were simply random, with scores based on weighted factors showing little advantage over unweighted ones.

The cumulative scores and dichotomous algorithms were compared on the basis of sensitivity, specificity, likelihood ratio (LR), positive predictive value $(\mathrm{PV}+)$, and "cost" (total proportion of the population referred). Table 2 shows the comparative utility of systems for cephalopelvic disproportion and post partum haemorrhage separately, in ascending order of cost. Because of the low incidences of these two conditions in this population, the positive predictive values are quite low, with none above $7 \%$. Focussing on nulliparous women who are less than $160 \mathrm{~cm}$ tall, however, would result in referrals of only $4 \%$ of all pregnant women and would identify $28 \%$ of the cephalopelvic disproportion cases. Using the presence of any two of the significant risk factors as the screening criteria achieves better sensitivity without too great a reduction in specificity and would result in just under $10 \%$ of women being referred and more than $40 \%$ of cephalopelvic disproportion cases being identified. Combining the strongest predictors, nulliparity (conditional on short stature) and operative delivery in the last pregnancy, yields nearly identical results in this population. At the high cost end, referring one quarter of the pregnant women (using the score with weighted factors and a cut off point
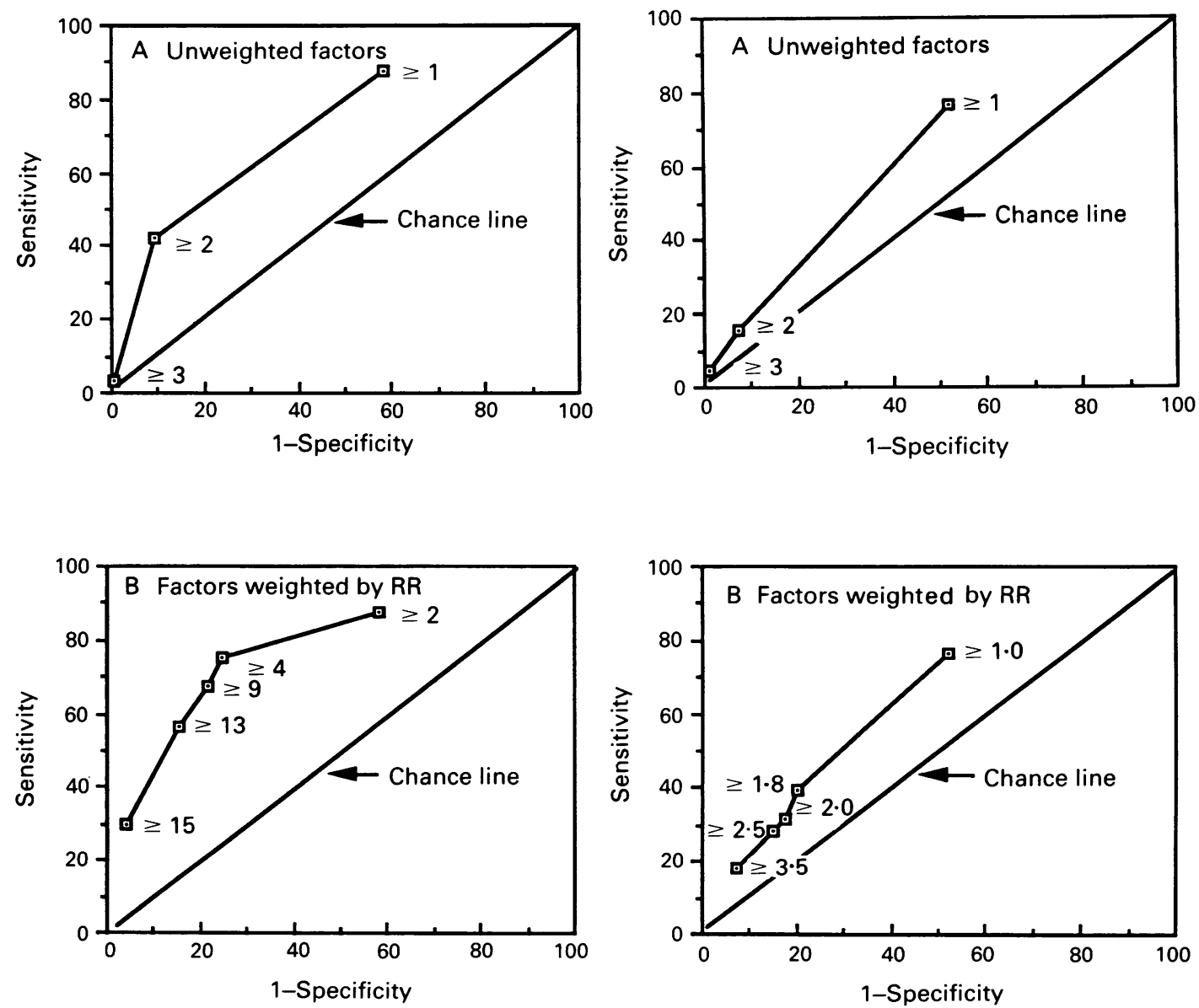

Figure 1 Receiver operating characteristic (ROC) curves for cephalopelvic disproportion based on cumulative scores using sums of unweighted risk factors and sums of risk factors weighted by the relative risk $(R R)$ values, at selected cut off points.

Figure 2 Receiver operating characteristic (ROC) curves for post partum haemorrhage based on cumulative scores using sums of unweighted risk factors and sums of risk factors weighted by the relative risk $(R R)$ values, at selected cut off points. 
of 4 or more) would result in three quarters of the cephalopelvic disproportion cases being predicted.

As was seen with the ROC curves, the post partum haemorrhage predictors are not as strong as those for cephalopelvic disproportion. The highest LR is achieved when the criteria consist of either poor obstetric outcome in the last pregnancy or antenatal admission to hospital for a pregnancy related problem. This combination identifies nearly a third of the cases, at a cost of only $5.6 \%$ of women being referred. Adding a low haemoglobin concentration to this combination increases

Table 2 Utility of various predictive criteria for cephalopelvic disproportion and post partum haemorrhage separately

\begin{tabular}{|c|c|c|c|c|c|}
\hline $\begin{array}{l}\text { Score system } \\
\text { (incidence of } \\
\text { condition) }\end{array}$ & $\begin{array}{l}\% \\
\text { predicted } \\
\text { (sensitivity) }\end{array}$ & Specificity & $\begin{array}{l}\text { Likelihood } \\
\text { ratio }\end{array}$ & $\begin{array}{l}\text { Predictive } \\
\text { value of } \\
\text { positive } \\
\text { test }(\%)\end{array}$ & $\begin{array}{l}\% \text { of } \\
\text { population } \\
\text { referred } \\
\text { ("cost") }\end{array}$ \\
\hline
\end{tabular}

(A) Cephalopelvic disproportion (.010):

(1) Nulliparous + height $<160$

(2) Any 2 or more risk factors

(3) (Nulliparous + height $<160$ ) or CPD $\mathrm{Hx}^{*}$

(4) \#3 or NND $\mathrm{Hx}^{\dagger}$ or PPH or (age $35+$ and height $<160$ )

(5) Nulliparous

6) Factors weighted by RR (cut $=13+$ )

\section{$28 \cdot 1$}

$42 \cdot 3$

40.9

$90 \cdot 5$

\section{$7 \cdot 0$}

$6 \cdot 6$

$4 \cdot 2$

(cut $=13+$ )
$\# 6(\mathrm{cut}=4+$

\section{$49 \cdot 8$}

$90 \cdot 0$

$$
4.5
$$

$4 \cdot 3$

$9 \cdot 8$

$\# 6(\mathrm{cut}=4+)$

\section{$53 \cdot 7$}

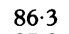

$4 \cdot 1$

$4 \cdot 0$

$10 \cdot 3$

$56 \cdot 2$
$72 \cdot 2$

$85 \cdot 3$

$3 \cdot 6$
$3 \cdot 7$

$3 \cdot 5$

$3 \cdot 6$

$14 \cdot 1$
$15 \cdot 1$

$15 \cdot 7$

$25 \cdot 3$

(1) Factors weighted by $R R$ (cut $=4+$ )

(2) ObHxił or antenatal hospitalisation

(3) Any 2 or more risk factors

(4) ObHx1† or antenatal hospitalisation or low haemoglobin

(5) \#1 (cut = 2+)

(6) Any 1 or more risk factor

$$
12 \cdot 7
$$

$30 \cdot 0$

$15 \cdot 4$

\section{$35 \cdot 0$}

35.0
31.5

$76 \cdot 5$

* CPD Hx: operative delivery in last pregnancy $+\mathrm{NND} \mathrm{Hx}$ : neonatal death in last pregnancy $\ddagger$ ObHx1: post partum haemorrhage, 1 st or 2 nd trimester fetal death, stillbirth, or neonatal death in last pregnancy.

Table 3 Utility of various predictive criteria for pooled outcomes (cephalopelvic

\begin{tabular}{|c|c|c|c|c|c|}
\hline $\begin{array}{l}\text { Score system } \\
\text { (incidence } \\
\text { of condition) }\end{array}$ & $\begin{array}{l}\% \\
\text { predicted } \\
\text { (sensitivity) }\end{array}$ & Specificity & $\begin{array}{l}\text { Likelihood } \\
\text { ratio }\end{array}$ & $\begin{array}{l}\text { Predictive } \\
\text { value of } \\
\text { positive test } \\
(\%)\end{array}$ & $\begin{array}{l}\% \text { of } \\
\text { population } \\
\text { referred } \\
\text { ("cost") }\end{array}$ \\
\hline $\begin{array}{l}\text { (A) Pooled }(\cdot 018) \text { : } \\
\text { (1) (Nulliparous }+ \text { height }<160) \\
\text { or ObHx2* or PIH } \\
\text { (2) Nulliparous } \\
\text { (3) Nulliparous }+ \text { height } \\
<160) \\
\text { or ObHx2* or antenatal } \\
\text { hospitalisation or } \\
\text { (age } 35+\text { or low } \\
\text { haemoglobin) }\end{array}$ & $\begin{array}{l}34 \cdot 5 \\
39 \cdot 0\end{array}$ & $\begin{array}{l}87 \cdot 6 \\
85 \cdot 3\end{array}$ & $\begin{array}{l}2 \cdot 8 \\
2 \cdot 7\end{array}$ & $\begin{array}{l}4 \cdot 9 \\
4 \cdot 7\end{array}$ & $\begin{array}{l}12 \cdot 8 \\
15 \cdot 1\end{array}$ \\
\hline $\begin{array}{l}\text { (B) Nulliparous }(\cdot 046) \text { : } \\
\text { (1) Antenatal } \\
\text { hospitalisation }\end{array}$ & $52 \cdot 2$ & $65 \cdot 2$ & 1.5 & $6 \cdot 7$ & $35 \cdot 0$ \\
\hline $\begin{array}{l}\text { (C) Muitiparas }(\cdot 013) \text { : } \\
\text { (1) ObHx2* } \\
\text { (2) ObHx2* or antenatal } \\
\text { hospitalisation }\end{array}$ & $\begin{array}{l}22 \cdot 5 \\
35 \cdot 3\end{array}$ & $\begin{array}{l}91 \cdot 0 \\
85 \cdot 3\end{array}$ & $\begin{array}{l}2 \cdot 5 \\
2 \cdot 4\end{array}$ & $\begin{array}{l}3 \cdot 2 \\
3 \cdot 1\end{array}$ & $\begin{array}{l}10 \cdot 1 \\
15 \cdot 3\end{array}$ \\
\hline
\end{tabular}
disproportion and post partum haemorrhage)

* ObHx2: operative delivery, neonatal death, or post partum haemorrhage in last pregnancy. the sensitivity to $35 \%$ but nearly doubles the cost to $10 \%$ of women being referred. Although the weighted score has a likelihood ratio of 4.2 at a cut off value of 4 or more, the sensitivity is so low as to be useless, and at a lower cut off of $2+(\mathrm{LR}=1 \cdot 8)$ it performs much worse than the simple combination of history and/or antenatal hospital admission. To identify three quarters of the post partum haemorrhage cases with these factors would require the referral of nearly half of all pregnant women.

Table 3 compares the predictive utility of these factors with regard to the two outcomes in a pooled population, as well as for pooled outcomes in two parity subgroups (nulliparas and multiparas). As one might expect, using the score systems for a pooled set of complications results in lower specificity and, thus, lower LRs. Nulliparity alone performed about as well as or better than any of the other more complicated algorithms, but it would have identified less than $40 \%$ of the cases complicated by either cephalopelvic disproportion or post partum haemorrhage. A combination of (1) shorter, nulliparous women, (2) those with an operative delivery, post partum haemorrhage, or neonatal death in the last pregnancy, and (3) those admitted to hospital for pregnancy induced hypertension during the current pregnancy yields a slightly higher LR than nulliparity alone, but results in a drop in sensitivity in exchange for its slightly reduced cost. Sensitivity can be increased to $42.4 \%$ by broadening the indications for antenatal hospital admission and adding older women with a low haemoglobin concentration, but specificity drops then and the cost climbs sharply. Even with all these limitations, in a pooled population the cost can be kept at about $13 \%$ of pregnant women being referred while predicting nearly $35 \%$ of the complicated deliveries.

The different incidence rates among the parity subgroups have a modest impact on the positive predictive values. For example, although antenatal hospital admission is neither very sensitive nor very specific among nulliparous women (evident in its low LR of 1.5), its positive predictive value is the highest of any of the score systems, primarily because the incidence of complications is so much higher in this population group. Despite the strength of obstetric history as a risk factor, its low sensitivity and the low rate of cephalopelvic disproportion and post partum haemorrhage among parious women greatly limit the positive predictive value of scoring systems in this group.

When individual probabilities for the complications of interest were estimated from the logistic models, actual cases did generally have higher probability scores than controls, but none of the probabilities was very high and there was considerable overlap between values for cases and controls (especially for post partum haemorrhage). For example, $80.6 \%$ of post partum haemorrhage cases and $92.2 \%$ of controls had estimated probabilities for post partum haemorrhage less than 0.02 . Since 
these probabilities are computationally more complex to generate and offer little advantage over simpler screening algorithms, no further evaluation of them is provided in this report.

\section{Discussion}

The value of these tests for antenatal screening clearly depends on the criteria by which they are judged. There is no single measure that satisfies all concerns. One of the main problems is that almost all measures are influenced by the characteristics of the population to which they are applied. Another is that both effectiveness and cost (in a very broad sense) must be taken into account.

\section{MEASURES OF UTILITY}

Sensitivity and specificity are basic measures of effectiveness that can characterise a test independently of the incidence of the target condition, but they can be affected by variations in the frequencies of the particular risk factors used. Attempts to combine sensitivity and specificity into one measure (called the "accuracy" or the "percent correctly classified"5) can be quite misleading ${ }^{10}$ and thus were not used here. The trade off between sensitivity and specificity was particularly evident in this study, since the sensitivity of individual factors was low, but more effective combinations of factors were inevitably less specific.

The low predictive values for all the screening tests in the present study are to be expected, at least to some extent, because of the low incidence of cephalopelvic disproportion and post partum haemorrhage in Harare. With an incidence of $2 \%$ or less, even a screening test with $95 \%$ sensitivity and $95 \%$ specificity could not achieve a positive predictive value better than $28 \%$. With regard to cephalopelvic disproportion, there is reason to believe (Munjanja, personal communication, 1991) that there might have been a somewhat higher incidence in Harare if women with two or more prior caesarean sections or other risk factors for cephalopelvic disproportion were not given elective caesareans, but even that adjustment would not push the incidence above $2 \%$. It is nearly impossible to get population based estimates of the incidence of cephalopelvic disproportion in Africa, but the figure for Harare is consistent with available data from other studies. ${ }^{11-15}$ While some hospital based studies have reported rates as high as $15 \%,{ }^{16}$ hospital populations usually over represent women with problems, a bias that is particularly true among "unbooked" patients (those not seen for antenatal care, who come to the hospital only when a complication occurs) and may also be true of "booked" patients, since these women may be self selected because of prior or anticipated delivery complications. It seems unlikely that the true underlying rate of cephalopelvic disproportion is much more than $2 \%$ in most places, so one cannot expect substantial improvement in predictive values for cephalopelvic disproportion in other populations.
There is some basis, though, for thinking that the incidence of post partum haemorrhage in Harare is considerably lower than the usual range of rates $(10-20 \%)$ reported elsewhere. ${ }^{17}$ This is partly a result of the exclusion of operative deliveries in this study and perhaps of the routine administration of syntometrine (a combination of oxytocin and ergometrine) after delivery to ensure prompt contraction of the uterus. Prendiville, Elbourne, and Chalmers, ${ }^{18}$ based on a review of data from controlled clinical trials, have estimated that active management of the third stage of labour (including the use of oxytocics) reduces the incidence of post partum haemorrhage by about $40 \%$. Begley ${ }^{19}$ compared post partum haemorrhage rates with and without oxytocics in a controlled trial in the UK and found a four fold higher rate without oxytocics $(8 \%$ versus $2 \%$ ). Such a ratio suggests a higher underlying rate of post partum haemorrhage in populations without intervention, very similar to the $8.4 \%$ rate found in a study of births attended by traditional birth attendants in Malawi. ${ }^{20}$ The rate of post partum haemorrhage in the Nigerian hospital series ${ }^{21}$ was $2 \cdot 8 \%$. If the more conservative correction factor of $40 \%$ held true in Harare, the incidence of post partum haemorrhage one could expect if oxytocics were not in use would be $1.3 \%$ instead of $0.8 \%$. If higher underlying rates of $8-10 \%$ were typical, some improvement in predictive values could be expected.

The lack of difference in predictive values between scores among nulliparous and parous women, despite the higher likelihood ratios for scores using obstetric history, is certainly partly due to the lower incidence of cephalopelvic disproportion and post partum haemorrhage in parous women. Alexander and Kierse $^{22}$ point out that although most studies find scoring to be more predictive in multiparous women, several exceptions to that have been reported previously. Also, scoring systems that rely more on socioeconomic factors generally find little difference in predictive value between nulliparous and parous women.

Cost considerations include the medical, financial, and social burdens associated with missed cases, unnecessary interventions, and administration of the test itself, as those burdens apply to individual women and their families and to the health care system. These costs are nearly impossible to quantify, but they can at least be compared on a relative basis to the current practice or to other screening options being considered. A major limitation on the usefulness of likelihood ratios and predictive values when making management decisions is that they give equal weight to false positives and false negatives, an approach that seldom reflects reality. In the present study, the costs of a missed case are high to the woman and the system, because both cephalopelvic disproportion and post partum haemorrhage are life threatening complications and are more expensive and difficult to manage if not treated promptly. The costs of unnecessary intervention, where intervention consists of referral for delivery in a facility where 
operative delivery and medical or surgical management of post partum haemorrhage are available, are also high in most parts of the developing world, where there are not enough personnel or facilities to accommodate all deliveries and many women cannot easily reach or afford care in an appropriate facility when the time for delivery arrives. Where health system capabilities are limited, unnecessary referrals may either overwhelm the system or pre-empt space needed for women who can truly benefit from hospital care. As was done in an earlier study in Zaire, ${ }^{23}$ this study defined cost as the percentage of women referred (that is, test positives) in the population. The costs of the tests themselves are negligible, given the type of screening systems discussed in this study (since they involve data already collected as part of routine physical examination and medical history taking), as long as antenatal care is already provided for purposes other than screening. Aside from haemoglobin determination, the factors are observational and not invasive.

Where the capacity of the health system is the limiting factor, screening tests must be evaluated with that consideration foremost. It is useful to choose a particular level of referrals that can be tolerated (Möller ${ }^{24}$ suggested $10-20 \%$ of women, for example) and then compare different tests on the basis of the proportion of cases they can detect within that referral limit. If referrals for hospital delivery were capped at $10 \%$ of pregnant women, a screening test that was considered positive if a women had two or more of the cephalopelvic disproportion risk factors present (test $(A) 2$ on table 2) would be the best and would detect $42.3 \%$ of cases. A screening test based on the presence of poor obstetric history, antenatal hospital admission for a pregnancy related problem, or haemoglobin less than $12 \mathrm{~g} / \mathrm{dl}$ (test (B) 4 on table 2) would be the best test for post partum haemorrhage within the $10 \%$ cap, but would detect only $35 \%$ of cases. None of the tests for a pooled outcome of cephalopelvic disproportion and post partum haemorrhage could meet the ceiling of $10 \%$ referred, although the screening test that combines short, nulliparous women, women with poor obstetric history, and women with pregnancy induced hypertension ( $(\mathrm{A}) 1$ on table 3 ) does not exceed it by much and identifies about $35 \%$ of cephalopelvic disproportion and post partum haemorrhage cases.

\section{COMPARISON WITH OTHER STUDIES OF}

OBSTETRIC SCREENING

While several studies in Africa have examined possible risk factors for obstetric complications, only a handful have looked systematically at the predictive values of the risk factors for maternal outcome. Two studies focussed on height and cephalopelvic disproportion. In Tanzania, primigravid women with heights of $146 \mathrm{~cm}$ or less accounted for $32 \%$ of the population and $90 \%$ of the caesarean sections for cephalopelvic disproportion ( $L R=3.0)$. If the cut off were set at $141 \mathrm{~cm}$ or less, only $11 \%$ of the population would be included while still identifying $67 \%$ of the cephalopelvic disproportion cases. ${ }^{11}$ Using a cut off of 60 inches or less $(150 \mathrm{~cm})$ for primigravidae in Sierra Leone, Aitken and Walls ${ }^{25}$ had to refer $57 \%$ of pregnant women in order to identify $84.6 \%$ of cephalopelvic disproportion cases $(\mathrm{LR}=0.5)$. Using the data from the present study and a cut off of $160 \mathrm{~cm}$ in nulliparous women, the predictive efficacy is midway between the Tanzania and Sierra Leone examples $(52.3 \%$ of cases detected for $14.9 \%$ of the population referred, $L R=1.9$ ).

Some studies have combined height with other factors. In Tanzania, a test with 18 possible factors ${ }^{26}$ identified $23.2 \%$ of pregnant women as "at risk" while detecting $54.3 \%$ of the cases of maternal complications at delivery $(\mathrm{LR}=2 \cdot 6)$. Also in Tanzania, pelvic assessment was used in primigravidae ${ }^{27}$ to detect $58.4 \%$ of cephalopelvic disproportion cases while referring just $21.8 \%$ for hospital delivery $(\mathrm{LR}=4 \cdot 2)$. In Zaire, two studies have linked risk factors with outcome. The Kasongo Project Team $^{23}$ compared risk factors recorded antenatally with two outcomes: life threatening fetopelvic disproportion and abnormally prolonged labour. They found that a history of previous dystocia or of previous perinatal deaths was significantly associated with both outcomes. They tested three approaches to risk prediction: (A) single factor, (B) any of several factors, and (C) specific combinations of factors. Among the single factors, a bad obstetric history was the most effective (sensitivity of $18 \%$ ) at the lowest cost (3\% referred). This is much better than a similar history factor in the current study, which identified only $16 \%$ of cephalopelvic disproportion cases with a cost of $9 \%$ referred. With model (B), using positivity for any one of several factors including a bad obstetric history, fetal position, and maternal age, height, and gravidity, the effectiveness rose to $69 \%$ of cases detected but cost rose to $44 \%$ of the population referred. With model (C), if either a bad obstetric history or young age with short height were present, effectiveness was $59 \%$ with a cost of $18 \%$ referred. In Karawa, Zaire, screening tests to identify those needing surgical delivery because of obstructed labour were based on parity, height, and obstetric history, but the most efficient test was based on height less than $145 \mathrm{~cm} .{ }^{16}$ By referring $13 \%$ of pregnant women, $46 \%$ of women with cephalopelvic disproportion could be identified. This is roughly comparable with the results achieved with test (A) 2 on table 2 (the presence of any two or more risk factors). At the high end of cost, if $26 \%$ of women were referred (for height $<150 \mathrm{~cm}$ ), $63 \cdot 7 \%$ of cephalopelvic disproportion cases were identified in Karawa. The use of height alone results in a slightly higher yield than when other factors are included, as in test (A)7 on table 2. This test, which consists of a score summing all risk factors, weighted by their RR values and using a cut off point of 4 or more, identifies nearly $18 \%$ more cases than the Karawa test $(75 \%$ versus $64 \%$ ) while referring the same propor- 
tion of pregnant women. The predictive values in the Karawa study are much higher than in the present study, though, because the incidence of cephalopelvic disproportion was so much higher in their hospital based sample. The Karawa study seems to be the only one published to date in which estimated probabilities (based on individual combinations of risk factors) using multiple logistic regression analysis have been calculated. Their derivation from a hospital based sample limits their application, but they do show a consistent trend in which the proportion of women with cephalopelvic disproportion is higher in groups with higher estimated probabilities.

Elsewhere in the developing world there have been some efforts to use risk factors to screen pregnant women. In Papua New Guinea, Lennox ${ }^{28}$ found $53 \%$ of women with both hospital and home based deliveries had at least one high risk factor and accounted for $70 \%$ of the complicated deliveries. A history of previous third stage of labour complications alone predicted $17 \%$ of subsequent third stage complications at a cost of just $3.5 \%$ of women referred. In a study in the Philippines, Esguerra $e t a l^{29}$ applied a score with antenatal and intrapartum components to a group of women and measured maternal and perinatal morbidity: while sensitivity was high, the specificity was low and the likelihood ratios were not much above $1 \cdot 0$. A Thai study ${ }^{30}$ tested a 43 item score against "abnormal deliveries" and achieved a sensitivity of $28.8 \%$, specificity of $84.4 \%$, and LR of 1.8 . Studies in other African settings ${ }^{31-35}$, in Australia, ${ }^{36}$ South America, ${ }^{37} 38$ and Asia ${ }^{39-41}$ describe risk screening programmes but fail to link risk status with actual outcome.

While risk systems in the developing world have dealt with both maternal and perinatal outcomes, those in the developed world deal almost exclusively with perinatal outcomes. (perhaps because poor maternal outcome is relatively rare). Several reviews of these systems have been published that point out the weaknesses and difficulties of the research done so far, including the absence of basic epidemiological statistics (such as sensitivity, specificity, or incidence rates) in many studies; the failure to acknowledge the potential impact of characteristics such as the age and ethnic distribution of particular study populations which limits their use in other groups; and the dependence of many systems on intrapartum information such as gestational age and birthweight (factors that are of little value in guiding management since they are detected too late for effective intervention). ${ }^{42-45}$

The ultimate value of any risk screening system depends not only on its effectiveness and cost but on the effectiveness of available interventions and the ability of the health system to implement them. In the cases of cephalopelvic disproportion and post partum haemorrhage, appropriate interventions (surgical and medical management) are well known and, at least in Zimbabwe, are available at most district hospitals. In places where such services are not available, any attempts to insti- tute risk screening programmes must be accompanied by plans to make the requisite interventions accessible to the population. For other obstetric complications, such as pregnancy induced hypertension or preterm labour, for which the interventions are more complex or less effective, the value of risk screening would be reduced.

\section{STUDY LIMITATIONS AND STRENGTHS}

There are several limitations inherent in this study and its setting that constrain the conclusions one can draw. One of the most significant is that the risk models were tested only on the data set from which they were drawn (as is true of the vast majority of such studies), a process that inflates their predictive values. ${ }^{22}$ An important follow up to this study would be to apply the screening tests to other data sets and see how well they do. Another limitation is the fact that the study is based on an urban population, even though the most urgent need for such risk screening is in the rural areas where ambulances and local hospitals are not available. This urban context may limit the extension of its findings to rural populations in Africa or other areas of the developing world. Although these women all had trained medical personnel attending their deliveries, a fact that distinguishes them from the vast majority of rural women, these deliveries involved a very low level of medical intervention, quite similar to the care rural women might receive. There is no obvious reason to believe that the risk factors identified in this study would differ for urban and rural residents. The common shifting of residence between urban and rural areas in Zimbabwe makes it less likely that Harare women differ substantially from their rural counterparts. If there were differences in the distribution of risk factors such as age or parity, they could affect the predictive values of the screening tests proposed. In particular, in a population where elective caesarean section because of a history of two or more previous sections is not common (unlike Harare, where it is official policy and nearly universally applied), the importance of nulliparity as a risk factor for cephalopelvic disproportion might drop considerably while the importance of a history of previous caesarean section might rise correspondingly. As a result, screening tests using nulliparity might be less effective while those using history might perform better.

A further limitation was the narrow focus of the study on two particular complicationscephalopelvic disproportion and post partum haemorrhage. Any useful antenatal screening system would have to address all possible serious obstetrical complications known to occur in significant numbers. In attempting to do so, the specificity of the test might decline somewhat (although this might be offset in terms of predictive value by a higher combined incidence rate). The more narrow systems described here may represent an overestimate of what can be achieved with risk screening. On the other hand, if the risk factors for cephalo- 
pelvic disproportion and post partum haemorrhage are also related to other obstetric and perinatal complications, the yield in terms of predictive value could be much higher in a combined screening test than in the ones shown here.

The conclusions to be drawn from this study regarding the value of risk screening are strengthened by several factors. Firstly, the screening tests were applied to groups representative of the general population of pregnant women in Harare, not just to a higher risk hospital population as in most other studies in Africa. The outcomes (cephalopelvic disproportion and post partum haemorrhage) were carefully defined and systematically ascertained from a uniform municipal medical record. The two outcomes were not subject to alteration as a result of early risk status identification, since they could only be treated and not prevented (except possibly for those women with two or more previous caesarean sections assigned to elective section for suspected cephalopelvic disproportion).

The results of this study suggest that a third or more of the women likely to experience cephalopelvic disproportion or post partum haemorrhage can be identified sufficiently in advance of the onset of labour to enable them to plan to deliver at a facility equipped to handle such problems. This can be done at a relatively modest cost, in terms of the testing itself and the excess of unnecessary referrals to the hospital, as long as antenatal care is already available or such screening can be incorporated into another community health care structure (such as traditional birth attendants or community health workers). Further refinements to increase the sensitivity or specificity of the screening tests or to determine risk factors that are common to other adverse outcomes (thereby increasing the pertinent incidence rates) would enhance the effectiveness of the tests. The continued expansion and improvement of the primary health care structure, including surgical services and blood transfusion capabilities relevant to other health problems besides obstetrics, would reduce the unit costs, both of administering the tests and of dealing with excess referrals, by broadening the service base and spreading it over several health functions. The screening models presented here provide a basis for comparing the value of screening for these conditions (cephalopelvic disproportion and post partum haemorrhage) with the results to be expected from alternative strategies, such as screening for other conditions of concern, treating symptomatic women only, or developing emergency transport or care capabilities in every community.

\section{Appendix}

The conditional probability $(\hat{\mathrm{p}})$ for an event (either cephalopelvic disproportion or post partum haemorrhage), given a set of values for the selected risk factors, was based on the fact that

$$
\hat{\mathbf{p}}=\mathrm{e}^{\operatorname{logit} \mathrm{p}} \div\left(1+\mathrm{e}^{\operatorname{logit} \mathrm{p}}\right)
$$

and for a case control study

$$
\begin{aligned}
\operatorname{logit} p= & \ln \left(P\left[\text { sampled } \mid{ }^{\text {case }}\right] \div P\left[{ }^{\text {sampled }} \mid{ }^{\text {control }}\right]\right) \\
& +\alpha+\beta_{1} x_{1}+\ldots+\beta_{k} x_{k}
\end{aligned}
$$

where alpha $(\alpha)$ represents the case-control intercept value, from which a sampling correction term - - $\left.\ln \left(P{ }^{\text {sampled }} \mid{ }^{\text {case }}\right] \div P\left[{ }^{\text {sampled }} \mid\right]\right)--$ must be added to get a cohort/based intercept, and each beta $(\beta)$ and $x$ represent a set of coefficients and factors. The numerator of the sampling correction term is 1 , since $100 \%$ of the cases were included. The proportion of controls sampled varied somewhat by facility of booking $(2 \cdot 3 \%$ of clinic, $0.6 \%$ of hospital, and $0.8 \%$ of unbooked). The logit was, therefore, adjusted by a different weighting factor depending on a given control's booking status.

I would like to express appreciation for the Fulbright Fellowship which supported the data collection in Zimbabwe and special thanks to Dr Noel S Weiss for his critical advice and constant encouragement throughout the research and preparation of this manuscript.

1 Adelstein P, Fedrick J. Antenatal identification of women at increased risk of being delivered of a low birth weight infant at term. Br f Obstet Gynaecol 1978;85:8-11.

2 Aubry RH, Nesbitt REL. High-risk obstetrics. Am $\mathcal{f}$ Obstet Gynecl 1969;105:241-7.

3 Goodwin JW, Dunne JT, Thomas BW. Antepartum identification of the fetus at risk. Can Med Assoc $\mathcal{F}$ 1969;101:458-64.

4 Hobel CJ. Recognition of the high-risk pregnant woman. In: Spellacy WN, ed. Management of the high-risk pregIn: Spellacy WN, ed. Management of the high-risk

5 Kaminski M, Goujard J, Rumeau-Rouquette C. Prediction of low birthweight and prematurity by a multiple regression analysis with maternal characteristics known since the beginning of pregnancy. Int $\mathcal{F}$ Epidemiol 1973;2:195-204.

6 Royston E, Lopez AD. On the assessment of maternal mortality. World Health Stat $Q$ 1987;40:214-24.

7 Tsu VD. Maternal height and age: Risk factors for cephalopelvic disproportion in Zimbabwe. Int $\mathcal{f}$ Epidemiol 1992;21:941-6.

8 Tsu VD. Postpartum haemorrhage in Zimbabwe: A risk factor analysis. Br $\mathcal{F}$ Obstet Gynaecol 1993;?:

9 Grant A, Mohide P. Screening and diagnostic tests in antenatal care. In: Enkin M, Chalmers I, eds. Effectiveness and satisfaction in antenatal care. London: William Heineman Medical, 1984; 22-59.

10 Weiss NS. Clinical epidemiology: The study of the outcome of illness. Monographs in epidemiology and biostatistics. Vol 1. Oxford: Oxford University Press, 1986; 21.

11 Everett VJ. The relationship between maternal height and cephalopelvic disproportion in Dar es Salaam. East African Med F 1975;52:251-6.

12 Fuchs K, Peretz, B-A, Marcovici R, Paldi E, Timor-Tritsh I. The 'grand multipara'. Is it a problem? A review of 5785 cases. Int $\mathcal{F}$ Gynecol Obstet 1985;23:321-5.

13 Howard D. Aspects of maternal morbidity: the experience of less developed countries. In: Jelliffe D, Jelliffe P, eds. Advances in international maternal and child health. Oxford: Clarendon, 1987; 1-35.

14 Liljestrand J, Bergstrom S, Westman S. Maternal height and perinatal outcome in Mozambique. 7 Trop Pediatr 1985;31:306-10.

15 Mtimavalye LAR, van der Does CD, Maathuis JB. The relationship between increasing birthweight and cephalopelvic disproportion in Dar es Salaam, Tanzania. $\mathcal{F}$ Obstet pelvic disproportion in Dar es Salaam, Tan

Gynaecol Br Commonwealth 1974;81:380-2.
16 Hermann CB, Duale S. Improving maternity care services in the Karawa Health Zone of Zaire. Maternal Nutrition and the Karawa Health Zone of Zaire. Maternal Nutrition and Health Care Program, Report No 2. International
for Research on Women: Washington DC, 1990.

17 World Health Organization (WHO). The prevention and management of post partum haemorrhage. Report of a Technical Working Group, WHO/MCH/90.7, Geneva: WHO, 1990.

18 Prendiville W, Elbourne D, Chalmers I. The effects of routine oxytocic administration in the management of the third stage of labour: An overview of the evidence from controlled trials. Br $\mathcal{F}$ Obstet Gynaecol 1988;95:3-16.

19 Begley CM. A comparison of 'active' and 'physiological' management of the third stage of labour. Midwifery 1990;6:3-17.

20 Bullough CHW, Msuku RS, Karonde L. Early suckling and post partum haemorrhage: Controlled trial in deliveries by traditional birth attendants. Lancet 1989;2: (8662):522-5.

21 Harrison KA. Background information. In: Harrison KA ed. Child-bearing, health and social priorities: a survey of 22,774 consecutive hospital births in Zaria, Northern Nigeria. Br f Obstet Gynaecol 1985;5(suppl):3-13.

22 Alexander S, Keirse MJNC. Formal risk scoring during 
pregnancy. In: Chalmers I, Enkin M, Kerise MJNC, eds. Effective care in pregnancy and childbirth. Vol 1. Oxford: Oxford University Press, 1989; 345-65.

23 Kasongo Project Team. Antenatal screening for fetopelvic dystocias: a cost-effectiveness approach to the choice of simple indicators for use by auxiliary personnel. $\mathcal{f}$ Trop Med Hyg 1984;87(4):173-83.

24 Moller B. The outcome of pregnancy and antenatal care in rural Tanzania. Acta Universitatis Upsaliensis, Comprehenrural Tanzania. Acta Universitatis Upsaliensis, Comprehensive summaries of Uppsala dissertations from the Facult

25 Aitken IW and Walls B. Maternal height and cephalopelvic disproportion in Sierra Leone. Trop Doct 1986;16:132-4.

26 Walther FJ. At-risk selection and outcome studies at the Kibara Hospital antenatal clinic in Tanzania. Trop Geogr Med 1980;32(4):336-9.

27 Bauer O, Kingu R, Laussen T, Mbwana K. Pelvic assessment and cephalopelvic disproportion in Central Tanzania. Int f Gynecol Obstet 1988;27:323-5.

28 Lennox CE. Assessment of obstetric high risk factors in developing country. Trop Doct 1984;14:125-9.

29 Esguerra AB, Diamante AN, Rams MM, et al. Concise screening scales for high-risk mothers and newborns. In: screening scalos $\mathrm{F}$. Primary maternal and neonatal health: A global concern. Primary maternal and neonatal healn

30 Sirivongs B, Parisunyakul S. Risk pregnancy screening: A simple method for non physicians to screen the high-risk simple method for non physicians to screen the high-r
pregnancy. $\mathcal{F}$ Med Assoc Thai $1984 ; 67$ (suppl) 2):15-20.

31 Essex BJ, Everett VJ. Use of an action-orientated record card for antenatal screening. Trop Doct 1977;7:134-8.

32 Sogbanmu MO. Perinatal mortality and maternal mortality in General Hospital, Ondo, Nigeria: Use of high-risk pregnancy predictive scoring index. Nigerian Med $\mathcal{f}$ 1979;9:475-9.

33 Malone MI. The quality of care in an antenatal clinic in Kenya. East African Med $\mathcal{f}$ 1980;57:86-89.
34 Kennedy I. Botswana: Midwives use antenatal cards to detect at-risk pregnancies. Salubritas 1982;6(4):1,7

35 Leke RJ, Nasah BT, Mtango FDE. Introduction of high risk pregnancy care in rural Cameroon: Health service research approach. Fournal of Obstetrics and Gynaecology East Central Africa 1988;7:7-10.

36 Watson DS. Use of "at risk" antenatal score card by aboriginal health workers in Arnhem Land. Trop Doct 1984;14:133-5.

37 Araujo G, Araujo L, Janowitz B, Wallace S, Potts $M$. Improving obstetric care in Northeast Brazil. Pan Amer Improving obstetric care in Northeast Brazil. Pan

38 Kirschbaum A, Salomon A, Parker E et al. Pregnancy risk factor assessment-North area of Santiago, Chile, 19821983. $M M W R$ 1985;34:335-7.

39 Shah KP. Surveillance card for married women for better obstetric performance. Fournal of Obstetrics and Gynaecology India 1978;28:1015-20.

40 Kumar V, Datta N. Home-based mothers' health records. World Health Forum 1988;9:107-10.

41 Alisjahbana A, Peeters R, Meheus A Traditional birth attendants can identify mothers and infants at risk. World Health Forum 1986;7:240-2.

42 Herman AAB, Irwig LM, Groeneveld HT. Evaluating obstetric risk scores by receiver operating characteristics obstetric risk scores by receiver operatin
curves. Am f Epidemiol 1988;127:831-42.

43 Marshall VA. A comparison of two obstetric risk assessment tools. 7 Nurse Midwifery 1989;34:3-7.

44 Morrison I, Carter L, McNamara S, Cheang M. A simplified intrapartum numerical scoring system: The prediction of high risk in labor. Am $\mathcal{f}$ Obstet Gynecol

45 Selwyn BJ. Review of obstetrical risk assessment methods In: Research issues in the assessment of birth settings. Washington, DC: National Academy Press, 1982; 149-70. 